\title{
Microscopic conditions favoring itinerant ferromagnetism
}

\author{
J. Wahle, ${ }^{*}$ N. Blümer, J. Schlipf, K. Held, and D. Vollhardt \\ Theoretische Physik III, Elektronische Korrelationen und Magnetismus, Universität Augsburg, D-86135 Augsburg, Germany
}

(Received 24 November 1997)

\begin{abstract}
A systematic investigation of the microscopic conditions stabilizing itinerant ferromagnetism of correlated electrons in a single-band model is presented. Quantitative results are obtained by quantum Monte Carlo simulations for a model with Hubbard interaction $U$ and direct Heisenberg exchange interaction $F$ within the dynamical mean-field theory. Special emphasis is placed on the investigation of (i) the distribution of spectral weight in the density of states, (ii) the importance of genuine correlations, and (iii) the significance of the direct exchange, for the stability of itinerant ferromagnetism at finite temperatures. We find that already a moderately strong peak in the density of states near the band edge suffices to stabilize ferromagnetism at intermediate $U$ values in a broad range of electron densities $n$. Correlation effects prove to be essential: Slater-Hartree-Fock results for the transition temperature are both qualitatively and quantitatively incorrect. The nearest-neighbor Heisenberg exchange does not, in general, play a decisive role. Detailed results for the magnetic phase diagram as a function of $U, F, n$, temperature $T$, and the asymmetry of the density of states are presented and discussed. [S0163-1829(98)08943-7]
\end{abstract}

\section{INTRODUCTION}

In contrast to conventional superconductivity and antiferromagnetism, metallic ferromagnetism is in general an intermediate or strong coupling phenomenon. Since there do not exist systematic investigation schemes to solve such types of problems the stability of metallic ferromagnetism is still not sufficiently understood. This is true even within the simplest electronic correlation model, the one-band Hubbard model, ${ }^{1}$ in spite of significant progress made recently. The Hubbard interaction is very unspecific, i.e., it does not depend on the lattice structure or dimension. Hence the dispersion, and thereby the shape of the density of states (DOS), is of considerable importance for the stability of ferromagnetism. This was recognized already by Gutzwiller, ${ }^{2}$ Hubbard, ${ }^{3}$ and $\mathrm{Kanamori}^{4}$ in their initial work on the Hubbard model. However, the approximations used in the early days of manybody theory were usually not reliable enough to provide definite conclusions. Exceptions are the exact results by Nagaoka $^{5}$ on the stability of ferromagnetism at $U=\infty$ in the case of one electron above or below half-filling. They show an important lattice sensitivity but, unfortunately, are not applicable in the thermodynamic limit.

Over the years the stability of metallic ferromagnetism has turned out to be a particularly difficult many-body problem whose explanation requires subtle nonperturbative techniques. There has been an upsurge of interest in this topic most recently. ${ }^{6-15}$ These investigations confirm that ferromagnetism is favored in systems with (i) frustrated lattices (which suppress antiferromagnetism) and (ii) high spectral weight near the band edge closest to the Fermi energy (which improves the kinetic energy of the polarized electrons). Taken together, these properties imply a strongly asymmetric DOS of the electrons. Ferromagnetism on bipartite lattices having a symmetric DOS may still be possible, but seems to require very large values of $U .{ }^{16}$ With the exception of Refs. 13 and 15 all previous calculations refer to the ground state. It is therefore of interest to obtain an answer to the question: How does the distribution of spectral weight in the DOS influence the stability regime of ferromagnetism at finite temperatures?

It should be noted that a strongly peaked, asymmetric DOS is a considerably more complex condition for ferromagnetism than the Stoner criterion. The latter merely asserts that, at $T=0$, the critical interaction for the instability is determined by the inverse of the DOS precisely at the Fermi energy $E_{F}, U_{c}=1 / N\left(E_{F}\right)$, thus neglecting antiferromagnetism and the structure of the DOS away from $E_{F}$. Stoner (i.e., Hartree-Fock ${ }^{17}$ ) theory is a purely static mean-field theory that ignores correlation effects, e.g., the correlationinduced redistribution of momentum states and the dynamic renormalizations of the band shape and width. So the question remains: How essential are genuine correlation effects for the stability of itinerant ferromagnetism at finite temperatures?

A third question concerns the suitability of the Hubbard model itself as a model for ferromagnetism. Indeed there is no compelling a priori reason why the Hubbard model should be a good model for ferromagnetism at all. Not only does it neglect band degeneracy, a feature observed in all ferromagnetic transition metals $(\mathrm{Fe}, \mathrm{Co}, \mathrm{Ni})$, it also ignores the (weak) direct Heisenberg exchange interaction that is equivalent to a ferromagnetic spin-spin interaction and hence favors ferromagnetism in the most obvious way. ${ }^{18-23}$ The proposition by Hirsch and co-workers ${ }^{19,20,22}$ that this interaction plays a key role in metallic ferromagnetism was disputed by Campbell et al. ${ }^{18}$ So the controversial question is: How important is the direct Heisenberg exchange interaction for the stability of itinerant ferromagnetism in the one-band Hubbard model at finite temperatures?

In this paper quantitative answers to the three questions formulated above are given within the dynamical mean field theory (DMFT). The DMFT, a nonperturbative approach, becomes exact in the limit of large coordination numbers. ${ }^{24-29}$ When applied to $d=3$, where the coordination number is $O(10)$, the DMFT has proven to yield accurate and reliable 
results, especially in the context of long-range magnetic order. $^{30,13}$ It treats local correlations exactly while spatial fluctuations are neglected. In this situation the momentum integral entering in the local propagator will be replaced by an energy integral involving only the DOS of the noninteracting electrons. The latter may be viewed as an input parameter. In our investigation the question concerning the importance of the distribution of spectral weight within the band for the stability of ferromagnetism will therefore be studied using a model DOS of the noninteracting electrons whose shape can be changed continuously from symmetric to strongly asymmetric by varying an asymmetry parameter.

The paper is structured as follows: in Sec. II we present the model under investigation, the dynamical mean-field equations, and the analytical and numerical steps needed to construct magnetic phase diagrams. The model DOS is introduced in Sec. III. The results of our investigation and quantitative answers to the questions posed above are presented in Sec. IV. A discussion where these results are put into perspective (Sec. V) closes the presentation.

\section{MODEL AND METHODS OF SOLUTION}

\section{A. Hubbard model with nearest-neighbor exchange}

The minimal model allowing one to treat an asymmetric DOS, electronic on-site correlations, and the nearestneighbor Heisenberg exchange interaction is given by

$$
\hat{H}=\hat{H}_{\mathrm{Hub}}-2 F \sum_{\langle i, j\rangle} \hat{\mathbf{S}}_{i} \cdot \hat{\mathbf{S}}_{j},
$$

where

$$
\hat{H}_{\mathrm{Hub}}=-\sum_{i, j, \sigma} t_{i j}\left(\hat{c}_{i \sigma}^{\dagger} \hat{c}_{j \sigma}+\text { H.c. }\right)+U \sum_{i} \hat{n}_{i \downarrow} \hat{n}_{i \uparrow} .
$$

Here $\hat{\mathbf{S}}_{i}=\frac{1}{2} \Sigma_{\sigma \sigma^{\prime}} \hat{c}_{i \sigma}^{\dagger} \boldsymbol{\tau}_{\sigma \sigma^{\prime}} \hat{c}_{i \sigma^{\prime}}$ with the vector of Pauli matrices $\tau$.

We note that there are three other nearest-neighbor contributions of the Coulomb interaction that might also affect the stability of the ferromagnetic phase ${ }^{31,32}$ (see Appendix).

\section{B. Dynamical mean-field theory}

Within the DMFT the coupling constants in Eqs. (1) and (2) have to be scaled with the lattice coordination number $Z$ as $^{24,25} t=t^{*} / \sqrt{Z}, F=F^{*} / Z$, where we consider nearestneighbor hopping $t$ only. By analogy to classical spin models $^{33}$ the Hartree-Fock approximation yields the exact result for the $F$ term in high dimensions.

In the following we investigate the influence of the direct exchange term on the properties of the Hubbard model in $d \rightarrow \infty$. Since the Hubbard model is SU(2) spin symmetric we can, without loss of generality, assume a magnetization parallel to the $z$ axis. The Hartree ${ }^{34}$ decoupling then takes the form $\hat{\mathbf{S}}_{i} \cdot \hat{\mathbf{S}}_{j} \rightarrow\left\langle\hat{S}_{i}^{z}\right\rangle \hat{S}_{j}^{z}+\hat{S}_{i}^{z}\left\langle\hat{S}_{j}^{z}\right\rangle-\left\langle\hat{S}_{i}^{z}\right\rangle\left\langle\hat{S}_{j}^{z}\right\rangle$. In terms of the magnetization $\hat{m}=\sum_{i} \hat{m}_{i} / N$ and its expectation value $m=\langle\hat{m}\rangle$, where $\hat{m}_{i}=2 \hat{S}_{i}^{z}=\hat{n}_{i \uparrow}-\hat{n}_{i \downarrow}$ and $N$ is the number of lattice sites, the Hamiltonian (1) can be written as ${ }^{35}$

$$
\hat{H}=\hat{H}_{\mathrm{Hub}}-\frac{N F^{*}}{2} m \hat{m}+\frac{N F^{*}}{4} m^{2} .
$$

Apparently the influence of the exchange term in the limit $d \rightarrow \infty$ is that of a (Weiss) magnetic field, which vanishes in the paramagnetic phase $(m=0)$. Therefore, in this phase, all one-particle properties of the system are those of the pure Hubbard model. However, two-particle functions, especially the ferromagnetic susceptibility, are modified (see Sec. II C).

In $d=\infty$, the Hubbard model (2) is equivalent to an Anderson impurity model complemented by a selfconsistency condition. ${ }^{36,29}$ Written in terms of Matsubara frequencies $\omega_{n}$, self-energy $\Sigma_{\sigma}$, the DOS of the noninteracting electrons $N^{0}(\varepsilon)$, and thermal average $\left\langle\psi \psi^{*}\right\rangle_{\mathcal{A}}$ over Grassmann fields the resulting coupled equations for the Green function in the homogeneous phase read

$$
\begin{gathered}
G_{\sigma}\left(i \omega_{n}\right)=\int_{-\infty}^{\infty} d \varepsilon \frac{N^{0}(\varepsilon)}{i \omega_{n}+\mu-\Sigma_{\sigma}\left(i \omega_{n}\right)-\varepsilon}, \\
G_{\sigma}\left(i \omega_{n}\right)=-\left\langle\psi_{\sigma n} \psi_{\sigma n}^{*}\right\rangle_{\mathcal{A}} .
\end{gathered}
$$

The solution of the $\boldsymbol{k}$-integrated Dyson equation (4) is straightforward and can be performed analytically for the DOS used in this paper (see Sec. IV). By contrast the solution of Eq. (5) is highly nontrivial (for details of the notation see Ref. 37). It is achieved using the auxiliary-field quantum Monte Carlo (QMC) algorithm by Hirsch and Fye, ${ }^{38}$ where a discretization of imaginary time, $\Delta \tau=\beta / \Lambda$, is introduced. Here, $\Lambda$ denotes the number of independent Matsubara frequencies. Physical quantities are obtained in the $\Delta \tau \rightarrow 0$ limit.

\section{Calculation of susceptibilities, extrapolation and error handling}

The second-order phase transition from a paramagnetic to a ferromagnetic phase occurs at the zero of the inverse susceptibility $\chi_{f}^{-1}$, calculated ${ }^{37,39}$ in the paramagnetic phase. It is sufficient to perform all simulations for the pure Hubbard model since the influence of $F^{*}$ on the susceptibility is given by the following random-phase approximation (RPA)-like expression:

$$
\chi_{f}\left(U, F^{*}, \ldots\right)=\frac{\chi_{f}(U, 0, \ldots)}{1-\left(F^{*} / 2\right) \chi_{f}(U, 0, \ldots)},
$$

where $\chi_{f}(U, 0, \ldots)$ is the susceptibility of the pure Hubbard model. This type of relation holds for pairs of two-particle interactions (here $U$ and $F^{*}$ ) in arbitrary dimensions, whenever one interaction (here $F^{*}$ ) is treated in Hartree-Fock approximation and the other one (here $U$ ) exactly. ${ }^{40}$ In general, since the calculation of the susceptibility involves the derivative of the self-energy $\Sigma_{U, F^{*}}$ with respect to some field $h,{ }^{37,39}$ this follows from the fact that the self-energy of the full Hamiltonian can be expressed as

$$
\Sigma_{U, F^{*}}\left[G_{U, F^{*}}\right]=\Sigma_{U, 0}\left[G_{U, F^{*}}\right]+\Sigma_{0, F^{*}}^{(1)}\left[G_{U, F^{*}}\right] .
$$

Here $\Sigma[G]$ refers to the diagrammatic skeleton expansion of $\Sigma$, where all lines are fully dressed propagators $G$. Since the Hartree-Fock term $\Sigma_{0, F^{*}}^{(1)}$ only contributes in the symmetry- 
broken phase, all $F^{*}$ renormalizations of $G_{U, F^{*}}$ vanish in the symmetric phase. Evaluating $d \Sigma_{U, F^{*}} / d h$ in the symmetric phase, the first term in Eq. (7) leads to the same contributions as without $F^{*}$ interaction, while the second term introduces the RPA-like term proportional to $F^{*}$. For the $t$ - $J$ model in DMFT the analog of Eq. (6) was derived by Pruschke et al. ${ }^{41}$

Since $F^{*}$ only enters the calculations via Eq. (7) we are left with four physical parameters of the pure Hubbard model: Hubbard interaction $U$, electron density $n$, temperature $T$, and an asymmetry parameter $a$ for the kinetic energy (see Sec. IV). For each set of these five parameters Eqs. (4) and (5) are iterated with typically $6 \times 10^{4}$ Monte Carlo sweeps until convergence is reached, i.e., the difference between two consecutive values of $\left(\boldsymbol{\mathcal { G }}_{\sigma}\right)^{-1}=\left(\boldsymbol{G}_{\sigma}\right)^{-1}-\boldsymbol{\Sigma}_{\sigma}$ is smaller than $5 \times 10^{-4}$ [measured by the norm $(2 \Lambda)^{-1} \Sigma_{\sigma n}\left|\left(\mathcal{G}_{\sigma n}^{\text {new }}\right)^{-1}-\left(\mathcal{G}_{\sigma n}^{\text {old }}\right)^{-1}\right|$; the energy scale is defined in Sec. IV]. Subsequently eight measurements of the susceptibility are performed with a reduced number of 2 $\times 10^{4}$ Monte Carlo sweeps. Thus the result for each parameter set consists of an averaged susceptibility $\chi_{f}(\Delta \tau)$ and its statistical error $\Delta \chi_{f}(\Delta \tau)$. We neglect the propagation of the error in $\left(\mathcal{G}_{\sigma}\right)^{-1}$ since it is always an order of magnitude smaller than $\Delta \chi_{f}(\Delta \tau)$. The extrapolation to $\Delta \tau=0$ is performed by a quadratic least-squares fit of $\chi_{f}(\Delta \tau)$, using at least six different values of $\chi_{f}$ for $\Delta \tau \in[0.09,0.5]$. Further details regarding the technical treatment can be found in Refs. 37 and 42.

For mean-field theories like the DMFT a linear behavior of the inverse susceptibility, i.e., a Curie-Weiss law, is expected and observed in the vicinity of the transition. Thus the Curie temperature $T_{C}$ can be obtained as the zero of a linear fit of $\chi_{f}^{-1}(T)$ drawn from values of $\chi_{f}$ for four to six different temperatures (see, e.g., Figs. 3 and 5, where $F_{c}^{*}=2 \chi_{f}^{-1}$ is plotted). The error of $T_{C}$ is obtained from the errors $\Delta \chi_{f}(\Delta \tau)$ by error propagation and therefore denotes only statistical, not systematic errors (e.g., due to the extrapolation schemes used). However, we checked the accuracy of our results by varying the procedure, e.g., extrapolating $\chi_{f}^{-1}(\Delta \tau)$ instead of $\chi_{f}(\Delta \tau)$.

\section{MODEL SPECTRAL FUNCTION}

Due to the vanishing of spatial fluctuations within the DMFT the topology of the underlying lattice enters the selfconsistency equation (4) only via the noninteracting DOS, at least for homogeneous phases. The choice of a particular model spectral function thus represents a special (not unique) set of hopping elements $t_{i j}$ in the Hamiltonian (1), which characterize the structure of the underlying lattice. Contributions to the kinetic energy by, e.g., next-nearest-neighbor hopping can lead to an asymmetrically shaped DOS, which is apparently favorable for the stability of ferromagnetism. To investigate this stabilizing effect quantitatively, we propose a model DOS with a shape-controlling parameter. This parameter allows us to change smoothly from a noninteracting DOS with (i) a symmetric shape (mimicking nearestneighbor hopping on a bipartite lattice) to (ii) an asymmetrically peaked DOS (similar to a cubic lattice with nextnearest-neighbor hopping) to (iii) a DOS with a square-root divergence at the band edge (e.g., a fcc lattice with nextnearest-neighbor hopping $t^{\prime}=t / 2$ ). The shape of the model

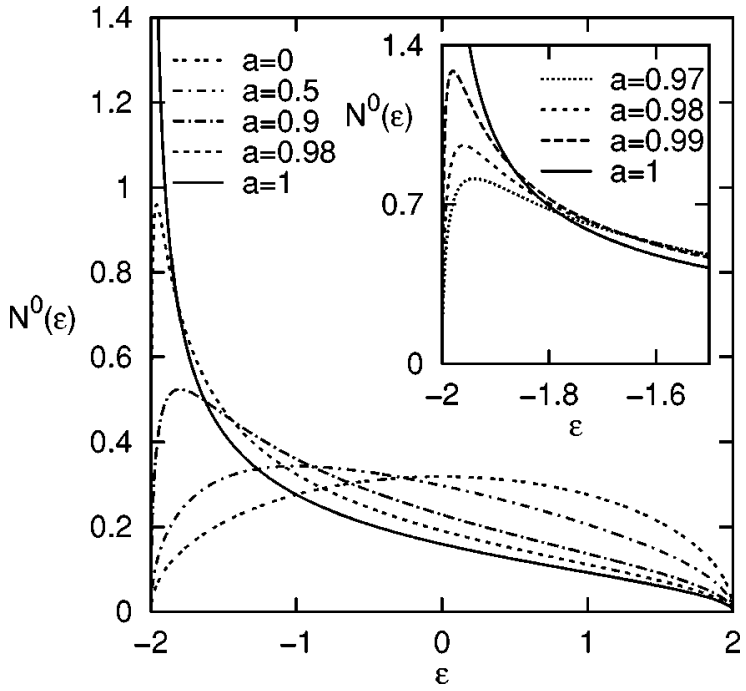

FIG. 1. Model spectral function (8) shown for different values of the asymmetry parameter $a$. By increasing $a$ spectral weight is shifted towards the lower band edge. The energy scale is fixed by setting the variance of the DOS equal to 1 .

DOS thus qualitatively captures key features of real lattices.

The spectral function that we use throughout the paper is given by

$$
N^{0}(\varepsilon)=c \frac{\sqrt{D^{2}-\varepsilon^{2}}}{D+a \varepsilon}
$$

with $c=\left(1+\sqrt{1-a^{2}}\right) /(\pi D)$ and bandwidth $2 D$. The wellknown semielliptic DOS of the Bethe lattice with infinite number of nearest neighbors is recovered for $a=0$. By increasing the parameter $a$ spectral weight is shifted towards the lower band edge (Fig. 1). For $a=1$ the DOS diverges at the lower band edge like an inverse square root. The particular choice of the model DOS (8) has the advantage that the numerical effort of solving the self-consistency equation (4) is rather small since the Hilbert transform can be calculated analytically. In the following we set the variance to unity, $\int d \varepsilon N^{0}(\varepsilon) \varepsilon^{2}-\left[\int d \varepsilon N^{0}(\varepsilon) \varepsilon\right]^{2}=1$, thereby fixing the energy scale. This leads to $D=2$ for all values of $a$. For $a$ $=0$ it is equivalent to choosing $t^{*}=1$ on the Bethe lattice.

While for the study of ferromagnetism within the DMFT the lattice structure only enters via the DOS it is possible to construct (infinitely many) corresponding dispersion relations $\varepsilon(\boldsymbol{k})$ or, equivalently, sets of hopping elements $t_{i j}$. A realization in $d=1$ that is symmetric, $\varepsilon(k)=\varepsilon(-k)$, and monotonous, $d \varepsilon / d k>0$, for $\varepsilon>0$ is given by

$$
\int_{\varepsilon_{\min }}^{\varepsilon} d \varepsilon^{\prime} N^{0}\left(\varepsilon^{\prime}\right)=\int_{0}^{k} \frac{d k^{\prime}}{\pi}=\frac{k(\varepsilon)}{\pi},
$$

where an inversion yields $\varepsilon(k)$. Generalizations to other dimensions are possible with, e.g., $\varepsilon(\boldsymbol{k})=\varepsilon(|\boldsymbol{k}|)$ (Ref. 43) or $\varepsilon(\boldsymbol{k})=\sum_{i=1}^{d} \varepsilon\left(k_{i}\right){ }^{44}$

Although in principle one could thus choose a lattice corresponding to the DOS (8), this will not be done here, since we only study homogeneous phases. Antiferromagnetism or incommensurate phases are not expected to be important far 


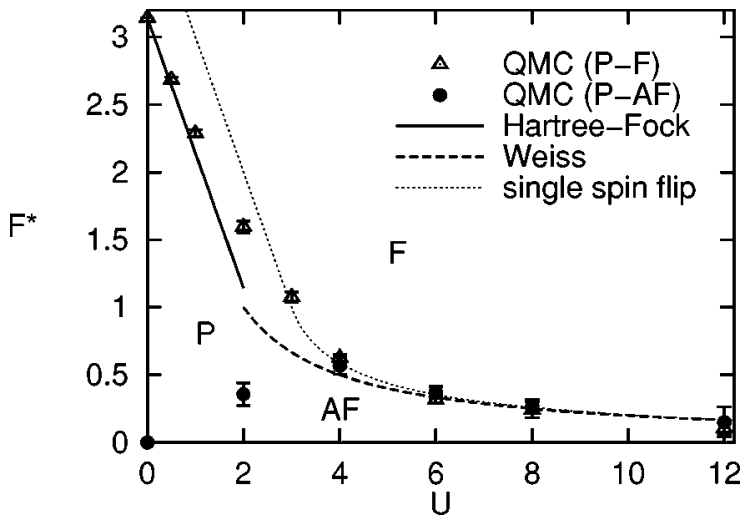

FIG. 2. Phase diagram for a symmetric DOS $(a=0)$ : direct exchange coupling $F^{*}$ vs Hubbard interaction $U$ at half filling ( $n$ $=1$ ) extrapolated to $T=0$. Open triangles and filled circles correspond to the instability of the paramagnetic phase $(P)$ against the ferromagnetic $(F)$ and antiferromagnetic $(\mathrm{AF})$ order, respectively. Solid line: Hartree-Fock theory; dashed line: Weiss mean-field theory; dotted line: single spin flip instability for the saturated ferromagnetic state.

away from half filling. Only for the case $a=0$ (Bethe lattice) at $n=1$ the stability of an antiferromagnetic phase is investigated.

\section{RESULTS}

\section{A. The importance of the direct exchange interaction}

On a bipartite lattice with perfect nesting, the ground state of the pure Hubbard model at half filling is antiferromagnetic for all $U>0$, at least in dimensions $d \geqslant 3$. In this situation a ferromagnetic state is strongly disfavored also in the general model, Eq. (1). At large $U$, however, when the model reduces to an effective Heisenberg model (which, in high dimensions, is exactly described by Weiss mean-field theory), already a small value of the direct exchange interaction, $F^{*}>2\left(t^{*}\right)^{2} / U$, is sufficient to stabilize a ferromagnetic ground state. ${ }^{45,46,21}$ Indeed, the Heisenberg model well describes the $F^{*}-U$ ground-state phase diagram at half filling down to $U \approx 4$; this is evident from Fig. 2, where a comparison with our QMC results is shown for a symmetric DOS [Eq. (8) with $a=0$ ]. At small $U$ the phase boundary between a paramagnetic and a ferromagnetic state is correctly reproduced by Hartree-Fock theory (but only for $U<1$ ). This is not surprising since in $d=\infty$ the $F$ term is treated exactly within this approximation. Also included in Fig. 2 is the line below which a fully saturated ferromagnetic state becomes unstable against single spin flips as first computed by Hirsch $^{47}$ for cubic lattices. For the Bethe lattice this line is given exactly by the Hartree-Fock result $F^{*}=4-U$ for $U$ $\leqslant U_{c}=3$ and

$$
F^{*}-U=-\frac{8}{\left(F^{*}+U\right)\left[1-\sqrt{1-16 /\left(F^{*}+U\right)^{2}}\right]}
$$

for $U>3$. This can be seen from Eqs. (5) and (7) in Ref. 47 and the known analytic expression for the Hilbert transform of the semielliptic DOS. The remarkable agreement between the QMC results and this curve for $U \geqslant 3$ suggests that (at

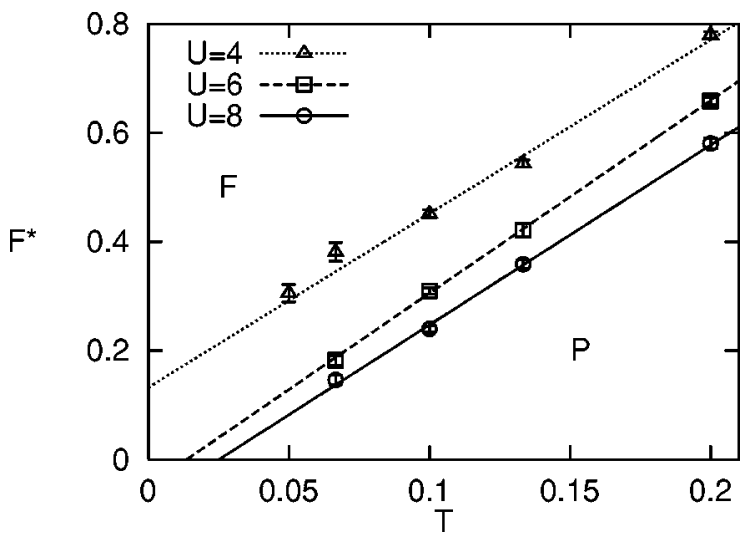

FIG. 3. $F^{*}-T$ phase diagram for different values of $U$ for a strongly peaked DOS $(a=0.98)$ at a filling $n=0.6$. The linear extrapolation shows that there exists a critical $U_{c}$ above which ferromagnetism is stable even without the direct exchange coupling.

zero temperature) the region of partial polarization is very narrow already at intermediate interaction strength $U$.

The $F^{*}-T$ phase diagram for a strongly peaked DOS ( $a=0.98$ ) at filling $n=0.6$ is shown in Fig. 3. The QMC results for the ferromagnetic phase boundary can be extrapolated linearly to zero temperature leading to a ground-state phase diagram. Clearly the values of $F^{*}$ necessary to stabilize ferromagnetism are significantly reduced in comparison to the bipartite case. In particular, for $U=6$ and $U=8$ the extrapolation lines cross the ordinate at positive temperatures. Thus, an asymmetric DOS stabilizes ferromagnetism even in the pure Hubbard model $\left(F^{*}=0\right)$ above a critical interaction strength $U_{c}$ with $4<U_{c}<6$.

Figures 3 and 4 prove that already a small direct exchange coupling $F^{*}$ can significantly enhance ferromagnetic tendencies and thus give the final "kick" towards ferromagnetism for systems that are close to an instability. This influence is stronger at larger densities when the local magnetic moments are enhanced (Fig. 4). The lower critical densities are very small, but larger than those predicted by Hartree-Fock theory, since Hartree-Fock always overestimates the size of the ferromagnetic regime.

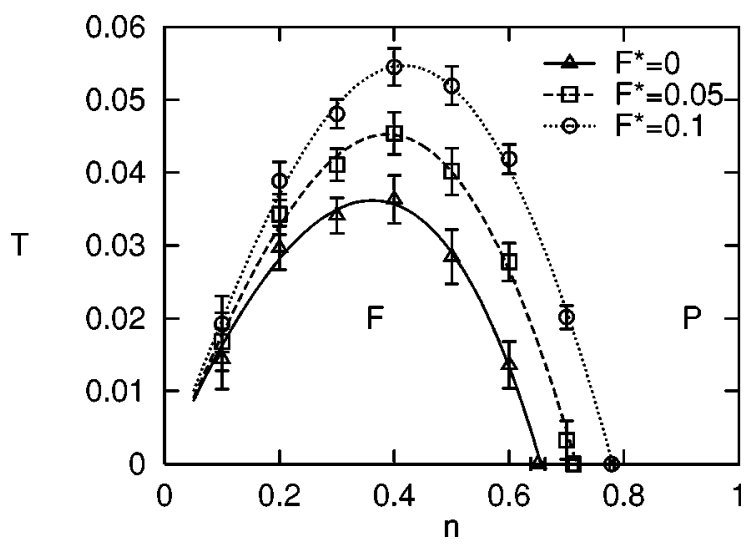

FIG. 4. $T-n$ phase diagram for different values of the direct exchange coupling $F^{*}$ for a strongly peaked DOS $(a=0.98)$ at $U$ $=6$. A small direct exchange coupling is seen to enlarge the stability regime of the ferromagnet, especially for densities close to half filling. The lines are a guide to the eye only. 


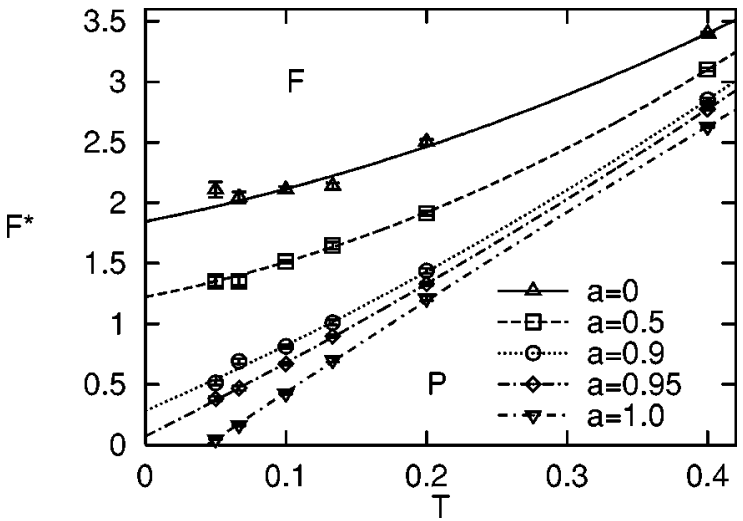

FIG. 5. $F^{*}-T$ phase diagram for different shapes of the DOS at $U=4$ and $n=0.3$. For values of $0.95<a \leqslant 1$ the ferromagnetic phase is stable even without the direct exchange coupling. The lines show a quadratic least-squares fit in $T$.

Quite generally, the value of the exchange interaction $F$ in metals can be expected to be rather small. Note, however, that for three-dimensional lattices the scaled quantity $F^{*}$ $=Z F$ is an order of magnitude larger than the exchange coupling $F$ itself. Hubbard's crude estimate ${ }^{3}$ of $F / U \approx 1 / 400$ would therefore imply that, e.g., at $U=6$ a (scaled) exchange interaction as large as $F^{*}=0.15$ is not completely unrealistic.

\section{B. The importance of the asymmetry of the DOS}

The dependence of the phase boundary on the asymmetry parameter $a$ is systematically studied in Fig. 5 at $U=4$ for a relatively small electron density $n=0.3$. For a symmetric or slightly asymmetric DOS $(a=0, a=0.5)$ the system only becomes ferromagnetic for $F^{*}>1$ even at $T=0$. For $a$ $=0.9$, when the shape of the DOS is roughly triangular, the critical $F^{*}$ is considerably reduced. But only when a marked peak develops (i.e., for $a>0.95$ ) does the critical $F^{*}$ drop to zero; ferromagnetism is then stable even in the pure Hubbard model. From now on we restrict our studies to this case $\left(F^{*}=0\right)$.

The $T$ versus $n$ phase diagram is shown in Fig. 6 for $U$ $=4$ and three different shapes of the DOS ranging from

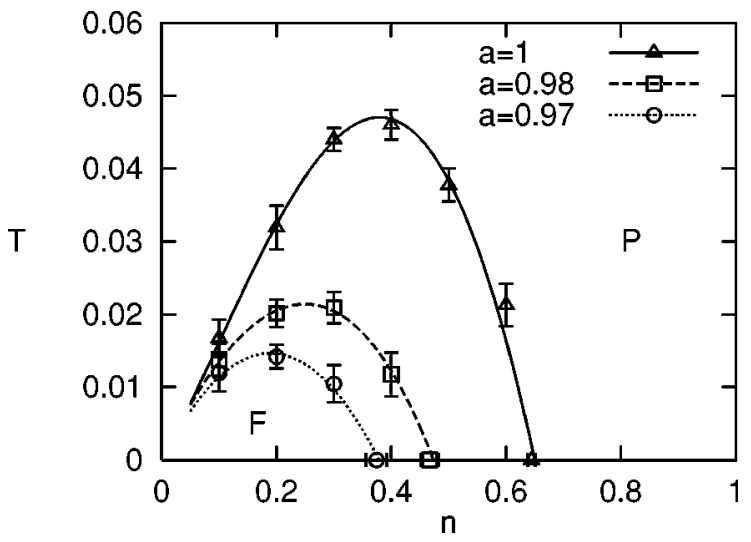

FIG. 6. $T-n$ phase diagram for different shapes of the DOS at $U=4$. By shifting spectral weight towards the lower band edge, the region of stability of the ferromagnetic phase is enlarged. The lines are a guide to the eye only.

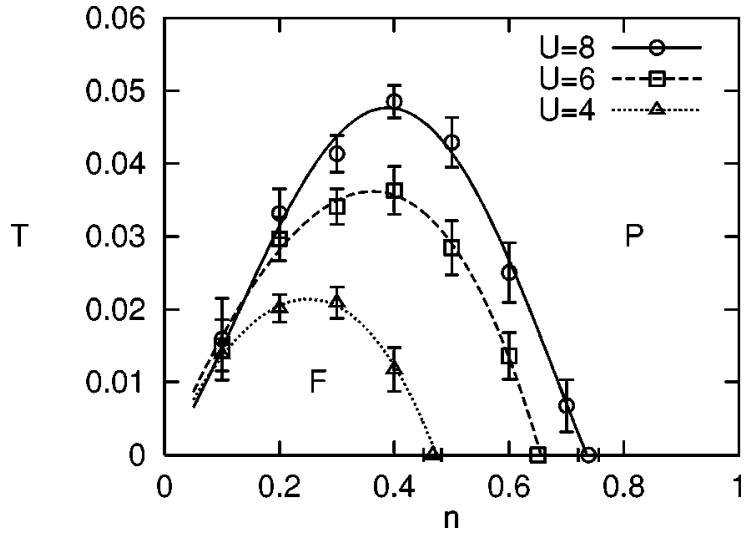

FIG. 7. $T-n$ phase diagram for a strongly peaked DOS ( $a$ $=0.98$ ) for different values of $U$. With increasing Hubbard interaction the stability regime of the ferromagnetic phase becomes larger, especially at higher densities. The lines are a guide to the eye only.

strongly asymmetric $(a=0.97)$ to divergent at the lower band edge $(a=1)$. Evidently the ferromagnetic phase is largest at $a=1$. We want to stress however, that the divergence does not change the physics qualitatively (except at $n \ll 1)$. A moderately strong peak near the band edge is all that is needed to stabilize ferromagnetism.

For symmetric densities of states at $U<12$ (Bethe lattice and hypercubic lattice) we find suppression of the ferromagnetic (as well as the antiferromagnetic) susceptibility away from half filling. ${ }^{48}$ This does not exclude the possibility for ferromagnetism on bipartite lattices at much larger values of $U$. Indeed, very recently ferromagnetism was found within the noncrossing approximation for the $t-J$ model on a hypercubic lattice in the limit $d \rightarrow \infty$ for $U>30$ away from half filling. ${ }^{16}$ Apparently, at least for moderate $U$, the bipartite lattice with only nearest-neighbor hopping is not a natural "environment" for ferromagnetism-the asymmetry of the noninteracting DOS is crucial.

\section{The importance of correlations}

In Fig. 7 the $T-n$ phase diagram is shown for different values of the on-site interaction $U$. Evidently the ferromagnetic phase becomes more favorable for increasing $U$ : both the maximal Curie temperature and the (upper) critical density rise. This effect is seen to be qualitatively similar to an increase of the exchange interaction $F^{*}$ or the asymmetry of the DOS $a$ (Figs. 4 and 6, respectively).

Our QMC results are compared with Hartree-Fock theory in Figs. 8-10. We note that, applied to the Hubbard model, the DMFT includes Hartree-Fock theory as its static limit and is thus superior in any dimension. Figure 8 shows the vast overestimation of the ferromagnetic phase within Hartree-Fock theory. The maximal Curie temperature obtained in this approximation is more than an order of magnitude too large. At such high temperatures details of the DOS are averaged out and consequently the density dependence (e.g., the position of the maximum) is completely artificial. In Fig. 9 the $U$ versus $n$ ground-state phase diagram is shown and compared to the Stoner criterion. ${ }^{17}$ At low $n$ the Stoner curve clearly approaches the QMC curve. Since the DOS vanishes smoothly at the lower band edge for $a<1$ 


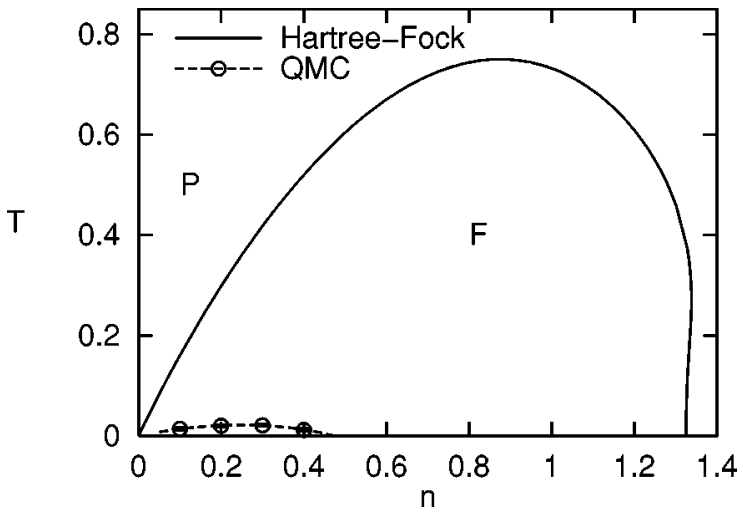

FIG. 8. T- $n$ phase diagram for a strongly peaked DOS ( $a$ $=0.98)$ at $U=4$. The comparison between Hartree-Fock theory (solid line) and DMFT (QMC, circles) reveals the importance of correlation effects. The dashed line is a guide to the eye only.

both curves diverge for $n \rightarrow 0$. Figure 10 focuses on the limit of large $U$. The weak coupling Hartree-Fock theory fails again: it predicts an unbounded linear increase of $T_{C}$ with $U$, $T_{C} \sim U n(2-n) / 4$, whereas $\mathrm{QMC}$ shows that $T_{C}$ has a finite limit for $U \rightarrow \infty$. It is expected that such a finite limit exists for all densities. A saturation is also suggested by the curves in Fig. 7. It arises from the suppression of double occupancies by correlations. In contrast to the Hartree-Fock prediction the interaction energy goes to zero for $U \rightarrow \infty$, thus only the bandwidth remains as an energy scale. In the special case $a=0.98, n=0.4$ one finds ${ }^{49} T_{C}(U=\infty)=0.07 \pm 0.02$.

One might argue that comparison of the DMFT results should not be made with Hartree-Fock itself but with Hartree-Fock plus quantum corrections, since the latter are known to reduce many of the deficiencies of Hartree-Fock theory. Such corrections have been discussed by van Dongen $^{50}$ and Freericks and Jarrell. ${ }^{51}$ The latter authors showed how quantum fluctuations modify the Stoner criterion by subtracting the particle-particle susceptibility. Evaluating these corrections in the case of Fig. 10 we find that the ferromagnetic phase is completely suppressed (as previously observed in Ref. 51 for a symmetric DOS). At $a=0.98$ this

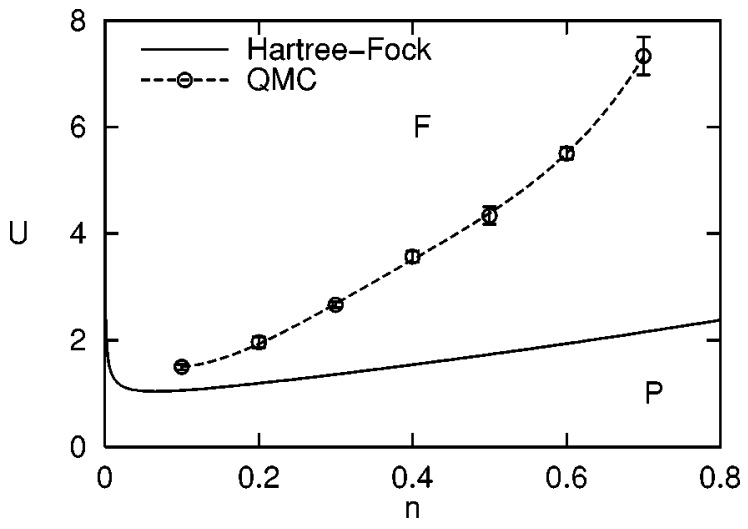

FIG. 9. $U-n$ phase diagram at $T=0$ for a strongly peaked DOS $(a=0.98)$. The DMFT data (QMC, circles) are extrapolated from finite temperature calculations. The Stoner criterion (solid line) underestimates the critical $U_{c}(n)$ for ferromagnetism, but becomes better at lower densities. The dashed line is a guide to the eye only.

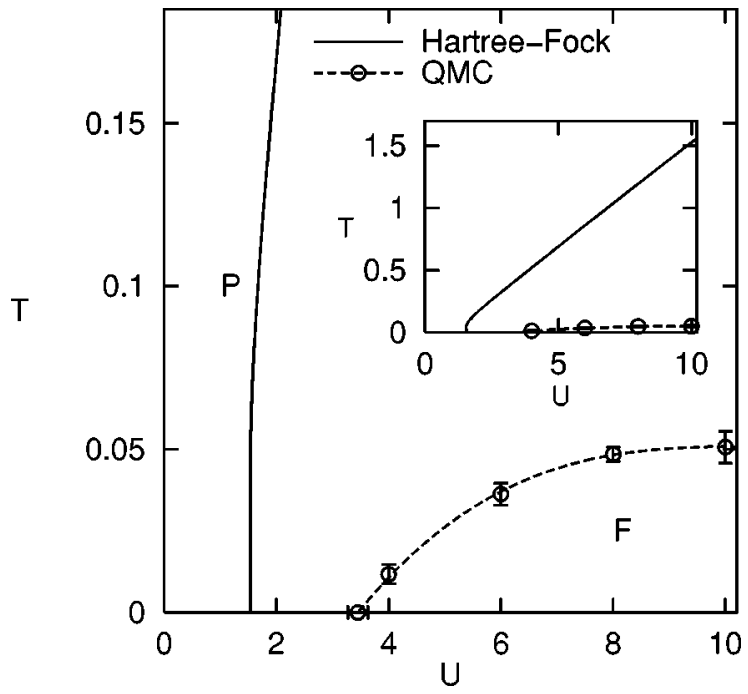

FIG. 10. $T-U$ phase diagram for a strongly peaked DOS ( $a$ $=0.98$ ) at $n=0.4$. The comparison between Hartree-Fock theory (solid line) and DMFT (QMC, circles) shows the former can describe the Curie temperature $T_{C}(U)$ neither quantitatively, nor qualitatively. The dashed line is a guide to the eye only.

holds for all densities $n \gtrsim 0.3$. Thus the "corrections" to Hartree-Fock theory are seen to underestimate the ferromagnetic region by far.

\section{DISCUSSION AND OUTLOOK}

After more than three decades of research it has become clear at last ${ }^{11-13}$ that the Hubbard model can describe itinerant ferromagnetism even on regular lattices and at moderate $U$ values for a wide range of electronic densities $n$. Since ferromagnetism is an intermediate to strong coupling problem the question concerning its "mechanism" has, in principle, no straightforward answer. This is in contrast to weak coupling phenomena, e.g., conventional superconductivity, which can be explained within perturbation theory. Nevertheless a good starting point for an understanding of the origin of itinerant ferromagnetism can be obtained in the strong coupling limit. At $U=\infty$ doubly occupied sites are excluded and the Hubbard model reduces to a (complicated) kinetic energy. To avoid doubly occupied sites in a paramagnetic phase the DOS is then necessarily strongly renormalized compared with the noninteracting case, whereas for the saturated ferromagnetic phase the interacting DOS is the same as the noninteracting one except for a shift between the spin-up and -down bands. In this situation details of the structure of the noninteracting DOS become relevant in selecting the state with the lowest energy. This physical picture is, in principle, similar to that underlying the Nagaoka mechanism; however, the latter only addresses the kinetic energy of a single hole and it was so far not possible to generalize it to thermodynamically relevant densities. Our investigations within the DMFT explicitly show that a moderately strong peak at the band edge closest to the Fermi energy is sufficient to stabilize ferromagnetism. Furthermore a strong asymmetry of the DOS implies a nonbipartite lattice that frustrates the competing antiferromagnetism near half filling.

The mechanism described above is completely different 
from the mere band shift of the Hartree-Fock theory. This weak coupling approach does not take into account the dynamical renormalization of the DOS in the paramagnetic phase and thus predicts ferromagnetism for any DOS, even at relatively small values of $U$ and for high temperatures. The comparison with DMFT clearly shows that Hartree-Fock theory (i) overestimates transition temperatures by more than an order of magnitude, (ii) renders the dependence of $T_{C}$ on $U$ qualitatively incorrect, and (iii) predicts ferromagnetism for the symmetric DOS, where (at least for $U<12$ ) this is not found. These shortcomings of Hartree-Fock theory are due to the neglect of dynamical fluctuations, which are at the heart of the correlation problem.

The Heisenberg exchange interaction, not considered in the pure Hubbard model, provides another mechanism that may order the fluctuating local moments arising by the suppression of double occupancies. We found that for a symmetric DOS rather large values of $F$ are needed to stabilize ferromagnetism. However, for an asymmetric DOS with a peak near the band edge already small values of the exchange interaction may provide the final "kick" towards ferromagnetism. In any case it reduces the critical on-site interaction and increases the critical temperatures of the ferromagnetic phase boundary.

While the DMFT correctly describes the dynamic fluctuations of the interacting many-body system, it neglects spatial fluctuations and short-range order. Hence one should suspect that this approach overestimates the transition temperatures $T_{C}$. Within DMFT Ulmke ${ }^{13}$ estimated $T_{C}$ for a threedimensional fcc lattice to be of the order of 500 to $800 \mathrm{~K}$, which is in the range of realistic transition temperatures. We may expect spatial fluctuations to reduce these temperatures. On the other hand, band degeneracy, not considered in our model so far, is expected to increase $T_{C}$. Indeed, band degeneracy and Hund's rule couplings, which are clearly present in realistic systems, can be rigorously shown to improve the stability of ferromagnetism at least for special parameter values. ${ }^{52,14}$ The incorporation of band degeneracy, for which the DMFT also provides a suitable framework, is the most important feature that has to be included in future investigations of the Hubbard model. ${ }^{53}$ The additional nearest-neighbor interactions discussed in the Appendix may provide yet another mechanism for ferromagnetism and will be studied in the future.

\section{ACKNOWLEDGMENTS}

We are very grateful to M. Kollar and M. Ulmke for numerous helpful discussions. One of us (N.B.) thanks the Fulbright Commission for support and the Department of Physics at the University of Illinois for hospitality. Computations were performed on the Cray T90 of the HLRZ Jülich.

\section{APPENDIX: NEAREST-NEIGHBOR INTERACTIONS}

In Wannier representation the Coulomb interaction gives rise to the purely local interaction $U$ as well as to four nearest-neighbor interactions. ${ }^{18-20,14}$ Besides the Heisenberg exchange interaction these are the density-density interaction, the pair-hopping term, and the off-diagonal "bond-charge-site-charge", 18 interaction. The latter effec-

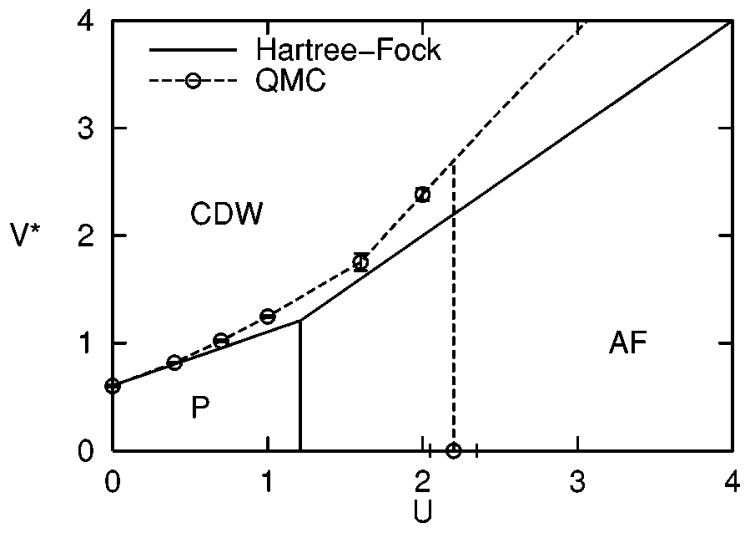

FIG. 11. $V^{*}-U$ phase diagram for the semielliptic DOS ( $a$ $=0$ ) at $T=0.125$. For $V^{*}>U$ a regime with charge-density-wave order (CDW) is established. The antiferromagnetic phase vanishes for $U>7.7 \pm 0.5$ (not shown). The dashed line is a guide to the eye only.

tively describes a density-dependent hopping ${ }^{20}$ and leads to a narrowing of the band. Hence this term is expected to stabilize saturated ferromagnetism. Since the quantum dynamics of this term make a systematic investigation difficult-even within the DMFT - its detailed study has to be postponed to the future. The pair-hopping term also weakly enhances ferromagnetic tendencies. ${ }^{20,32}$

Among all nearest-neighbor interactions the densitydensity term

$$
\hat{H}_{\mathrm{NN}}^{V}=V \sum_{\langle i, j\rangle} \hat{n}_{i} \hat{n}_{j}
$$

is largest and is thus investigated explicitly in the following. In the case of $d$ electrons Hubbard roughly estimated this term to $V=2-3 \mathrm{eV}$, an order of magnitude smaller than the Hubbard interaction $U{ }^{3}$ However, since there are $Z$ neighbors contributing, the total energy of the nearest-neighbor density-density interactions may in some materials even surpass that of the Hubbard interaction. This raises the question of the importance of the $V$ term, in particular its influence on the ferromagnetic phases investigated in the present paper.

It was already pointed out by Müller-Hartmann ${ }^{25}$ that in the limit $d \rightarrow \infty$ and with the proper scaling $V=V^{*} / Z$ the nearest-neighbor density-density interaction reduces to its Hartree contribution, which may then be viewed as a simple, site-dependent shift of the chemical potential. In the absence of broken translational symmetry the chemical potential must compensate this shift to keep the electron density fixed. Then there is no effect at all.

On bipartite lattices translational symmetry can be broken by a charge-density wave (CDW) with different electron densities on $A$ and $B$ sublattices, i.e., with order parameter $n_{\mathrm{CDW}}=\left(n_{A}-n_{B}\right) / 2$. To study this possible ordering we analyze the instability towards a CDW in the following.

Similar to the exchange term $F$ the Hartree contribution of the interaction $V$ leads, even in the presence of other interactions, to an RPA-like pole in the CDW susceptibility [cf. Eq. (6)]: ${ }^{40}$

$$
\chi_{\mathrm{CDW}}\left(U, V^{*}, \ldots\right)=\frac{\chi_{\mathrm{CDW}}(U, 0, \ldots)}{1-V^{*} \chi_{\mathrm{CDW}}(U, 0, \ldots)} .
$$


Thus a second-order phase transition to a CDW occurs at $V_{c}^{*}=1 / \chi_{\mathrm{CDW}}(U, 0, \ldots)$.

Since next-nearest-neighbor hopping frustrates CDW order, the maximal instability towards a CDW is expected for the symmetric DOS with $a=0$ in Eq. (8). Half filling is optimal in this case. For these parameters we determined the phase diagram Fig. 11 employing the QMC technique (for details concerning the calculation of the CDW susceptibility see Ref. 54). Within DMFT a CDW ordering occurs for $V^{*} \gtrsim U$ (at not too high temperatures). Compared to the Hartree-Fock approximation the CDW phase boundary of the full model is only slightly moved towards larger values of $V^{*}$. A similar deviation from Hartree-Fock theory was found by means of QMC simulations in $d=1$ by Hirsch, ${ }^{55}$ in $d$ $=2$ by Zhang and Callaway, ${ }^{56}$ and within perturbation theory for both weak and strong coupling (in arbitrary dimensions) by van Dongen. ${ }^{50,57}$
All these studies demonstrate that the CDW is stable for $V^{*}=V Z>U$. While this relation may in principle hold for some transition metals, ferromagnets apparently do not show spatial charge ordering. Therefore the adequate correlated electron model for a ferromagnet appears to have parameters in the range $V^{*} \leqslant U$. Then the nearest-neighbor densitydensity interaction $V^{*}$ has no influence on the phase diagram, especially on the border of the ferromagnetic phase, at least in $d=\infty$. Even in $d=3$ the Hartree diagram gives the main contribution of the interaction $V^{*}$ since spatial fluctuations, leading to genuine correlations, are suppressed as $1 / Z$. Moreover in $d=1$ and at half filling the effect of $V^{*}$ on the ferromagnetic phase boundary is still small. ${ }^{22}$ Therefore, over an extended range of parameters the nearest-neighbor term $V^{*}$ has almost no influence and thus its importance is seen to be much smaller than its value suggests.
*Present address: Theoretische Physik, Universität Duisburg, Lotharstr. 1, D-47048 Duisburg, Germany.

${ }^{1}$ It is known, however, that in the one-dimensional Hubbard model with only nearest-neighbor hopping ferromagnetism is unstable [E. Lieb and D. C. Mattis, Phys. Rev. 125, 164 (1962)].

${ }^{2}$ M. C. Gutzwiller, Phys. Rev. Lett. 10, 59 (1963).

${ }^{3}$ J. Hubbard, Proc. R. Soc. London, Ser. A 276, 238 (1963).

${ }^{4}$ J. Kanamori, Prog. Theor. Phys. 30, 275 (1963).

${ }^{5}$ Y. Nagaoka, Phys. Rev. 147, 392 (1966).

${ }^{6}$ E. H. Lieb, in The Hubbard Model: Its Physics and Mathematical Physics, edited by D. Baeriswyl et al. (Plenum, New York, 1995), p. 1; P. Fazekas, Philos. Mag. B 76, 797 (1997).

${ }^{7}$ A. Mielke, J. Phys. A 24, 3311 (1991); H. Tasaki, Phys. Rev. Lett. 69, 1608 (1992).

${ }^{8}$ K. Penc, H. Shiba, F. Mila, and T. Tsukagoshi, Phys. Rev. B 54, 4056 (1996).

${ }^{9}$ E. Müller-Hartmann, in Proceedings of the V. Symposium on Physics of Metals, edited by E. Talik and J. Szade (Symposium “'Physics of Metals,', Ustron-Jaszowiec, Poland, 1991), p. 22; E. Müller-Hartmann, T. Hanisch, and R. Hirsch, Physica B 186188, 834 (1993); T. Hanisch and E. Müller-Hartmann, Ann. Phys. (Leipzig) 2, 381 (1993); T. Hanisch, B. Kleine, A. Ritzl, and E. Müller-Hartmann, ibid. 4, 303 (1995); P. Wurth, G. S. Uhrig, and E. Müller-Hartmann, ibid. 5, 148 (1996); T. Hanisch, G. S. Uhrig, and E. Müller-Hartmann, Phys. Rev. B 56, 13960 (1997).

${ }^{10}$ G. S. Uhrig, Phys. Rev. Lett. 77, 3629 (1996).

${ }^{11}$ S. Daul and R. Noack, Z. Phys. B 103, 293 (1997).

${ }^{12}$ R. Hlubina, S. Sorella, and F. Guinea, Phys. Rev. Lett. 78, 1343 (1997).

${ }^{13}$ M. Ulmke, Eur. Phys. J. B 1, 301 (1998).

${ }^{14}$ D. Vollhardt, N. Blümer, K. Held, M. Kollar, J. Schlipf, and M. Ulmke, Z. Phys. B 103, 283 (1997).

${ }^{15}$ T. Herrmann and W. Nolting, J. Magn. Magn. Mater. 170, 253 (1997); Solid State Commun. 103, 351 (1997); A. Vega and W. Nolting, Phys. Status Solidi B 193, 177 (1996).

${ }^{16}$ T. Obermeier, T. Pruschke, and J. Keller, Phys. Rev. B 56, R8479 (1997).

${ }^{17}$ For ferromagnetism in the Hubbard model, Hartree-Fock theory is equivalent to Stoner theory.

${ }^{18}$ D. K. Campbell, J. T. Gammel, and E. Y. Loh, Jr., Phys. Rev. B 38, 12043 (1988).
${ }^{19}$ J. E. Hirsch, Phys. Rev. B 40, 2354 (1989).

${ }^{20}$ J. E. Hirsch, Phys. Rev. B 40, 9061 (1989); S. Tang and J. E. Hirsch, ibid. 42, 771 (1990).

${ }^{21}$ R. Strack and D. Vollhardt, J. Low Temp. Phys. 99, 385 (1995).

${ }^{22}$ J. C. Amadon and J. E. Hirsch, Phys. Rev. B 54, 6364 (1996).

${ }^{23}$ M. Kollar, R. Strack, and D. Vollhardt, Phys. Rev. B 53, 9225 (1996).

${ }^{24}$ W. Metzner and D. Vollhardt, Phys. Rev. Lett. 62, 324 (1989).

${ }^{25}$ E. Müller-Hartmann, Z. Phys. B 74, 507 (1989).

${ }^{26}$ E. Müller-Hartmann, Int. J. Mod. Phys. B 3, 2169 (1989).

${ }^{27}$ V. Janiš, Z. Phys. B 83, 227 (1991); V. Janiš and D. Vollhardt, Int. J. Mod. Phys. B 6, 731 (1992).

${ }^{28}$ D. Vollhardt, in Correlated Electron Systems, edited by V. J. Emery (World Scientific, Singapore, 1993).

${ }^{29}$ A. Georges, G. Kotliar, W. Krauth, and M. J. Rozenberg, Rev. Mod. Phys. 68, 13 (1996).

${ }^{30}$ M. Jarrell and T. Pruschke, Z. Phys. B 90, 187 (1993); M. J. Rozenberg, G. Kotliar, H. Kajüter, G. A. Thomas, D. H. Rapkine, J. M. Honig, and P. Metcalf, Phys. Rev. Lett. 75, 105 (1995); M. J. Rozenberg, I. H. Inoue, H. Makino, F. Iga, and Y. Nishihara, ibid. 76, 4781 (1996); M. Ulmke, R. T. Scalettar, A. Nazarenko, and E. Dagotto, Phys. Rev. B 54, 16523 (1996).

${ }^{31}$ H. Q. Lin and J. E. Hirsch, Phys. Rev. B 52, 16155 (1995).

${ }^{32}$ J. E. Hirsch, Phys. Rev. B 43, 705 (1991).

${ }^{33}$ See, e.g., C. Itzykson and J. M. Drouffe, Statistical Field Theory (Cambridge University Press, Cambridge, 1989).

${ }^{34}$ The Fock contribution of the $F$ term vanishes as $1 / Z$ since it is given by two Green functions connecting neighboring sites. In $d=3$ the Fock term renormalizes the band-width: $\widetilde{D}(E(\epsilon))$ $=D(\epsilon) /\left\{1-F^{*} /\left(Z t^{* 2}\right) \int d \epsilon D(\epsilon)(-2 \epsilon) f[E(\epsilon)]\right\}$ [see Eq. (29) in Ref. 19, which readily yields this equation for the paramagnetic phase]. The renormalization is maximal at half-filling and reduces the critical value of $F^{*}$ for the onset of ferromagnetism, e.g., for the half-filled cubic lattice from $F^{*}=1.17 t$ to $F^{*}$ $=0.84 t$ within the Hartree-Fock approximation $\left(F^{*} \widetilde{D}\left(\epsilon_{F}\right)\right.$ $=1)$. This effect of order $1 / Z$ is neglected in the DMFT.

${ }^{35}$ The definition of generalized (e.g., staggered) susceptibilities for bipartite $(A B-)$ lattices requires the introduction of an index $\alpha$ $\in\{A, B\}$ in Eq. (3) that indicates the sublattice. The corresponding generalizations are straightforward (see, e.g., Ref. 39).

${ }^{36}$ M. Jarrell, Phys. Rev. Lett. 69, 168 (1992); A. Georges and G. 
Kotliar, Phys. Rev. B 45, 6479 (1992).

${ }^{37}$ M. Ulmke, V. Janiš, and D. Vollhardt, Phys. Rev. B 51, 10411 (1995).

${ }^{38}$ J. E. Hirsch and R. M. Fye, Phys. Rev. Lett. 56, 2521 (1986).

${ }^{39}$ K. Held, M. Ulmke, N. Blümer, and D. Vollhardt, Phys. Rev. B 56, 14469 (1997).

${ }^{40}$ J. Schlipf and M. Kollar (unpublished).

${ }^{41}$ T. Pruschke, Q. Qin, T. Obermeier, and J. Keller, J. Phys.: Condens. Matter 8, 3161 (1996).

${ }^{42}$ M. Ulmke, Doctoral thesis, KFA Jülich, 1995.

${ }^{43}$ M. Kollar (private communication).

${ }^{44} \mathrm{M}$. Ulmke (private communication).

${ }^{45}$ D. K. Campbell, J. T. Gammel, and E. Y. Loh, Jr., Phys. Rev. B 38, 12043 (1988).

${ }^{46}$ S. Tang and J. E. Hirsch, Phys. Rev. B 42, 771 (1990).

${ }^{47}$ J. E. Hirsch, J. Appl. Phys. 67, 4549 (1990).
${ }^{48}$ N. Blümer, Diploma thesis, RWTH Aachen, 1996.

${ }^{49}$ The error mainly reflects an uncertainty in the extrapolation law. It was determined by comparing linear and quadratic leastsquares fits in $1 / U$.

${ }^{50}$ P. G. J. van Dongen, Phys. Rev. Lett. 67, 757 (1991).

${ }^{51}$ J. K. Freericks and M. Jarrell, Phys. Rev. Lett. 74, 186 (1995).

${ }^{52}$ M. Kollar and D. Vollhardt (unpublished).

${ }^{53}$ K. Held and D. Vollhardt, cond-mat/9803182, Eur. Phys. J. B (to be published).

${ }^{54}$ K. Held, M. Ulmke, and D. Vollhardt, Mod. Phys. Lett. B 10, 203 (1996).

${ }^{55}$ J. E. Hirsch, Phys. Rev. B 28, 4059 (1983).

${ }^{56}$ Y. Zhang and J. Callaway, Phys. Rev. B 39, 9397 (1989).

${ }^{57}$ P. G. J. van Dongen, Phys. Rev. B 49, 7904 (1994); 50, 14016 (1994). 